\title{
Italique
}

Poésie italienne de la Renaissance

I| 1998

Varia

\section{Minturno, Marino e un modulo oraziano}

\section{Stefano Carrai}

\section{OpenEdition}

\section{Journals}

Edizione digitale

URL: http://journals.openedition.org/italique/91

DOI: 10.4000/italique.91

ISSN: 1663-4438

\section{Editore}

Librairie Droz

\section{Edizione cartacea}

Data di pubblicazione: 1 giugno 1998

Paginazione: 95-101

ISBN: 2-600-00239-1

ISSN: 1423-3983

Notizia bibliografica digitale

Stefano Carrai, " Minturno, Marino e un modulo oraziano », Italique [Online], I 1 1998, online dal 14 septembre 2009, consultato il 30 avril 2019. URL : http://journals.openedition.org/italique/91 ; DOI : 10.4000/italique. 91 
STEFANO CARRAI

MINTURNO, MARINO

E UN MODULO ORAZIANO 


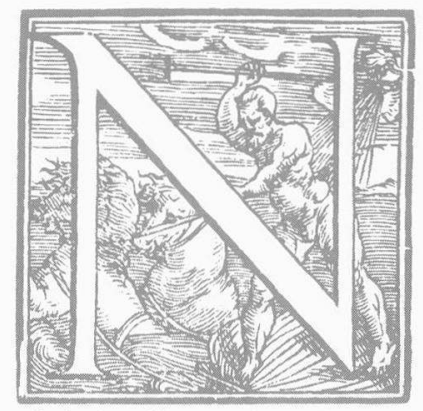

EL sonetto proemiale delle Rime amorose, primo libro della vasta raccolta pubblicata nel 1602, Marino, coerentemente con l'argomento, enunciava il programma di astenersi dal genere eroico per dedicarsi al canto esclusivo dell'amore. L'opposizione acquisiva risalto per effetto dello schematico andamento delle due quartine. Nella prima il poeta formulava la rinuncia, in favore d'altri, alla poesia epica e nella seconda dichiarava la propria dedizione verso quella amorosa:

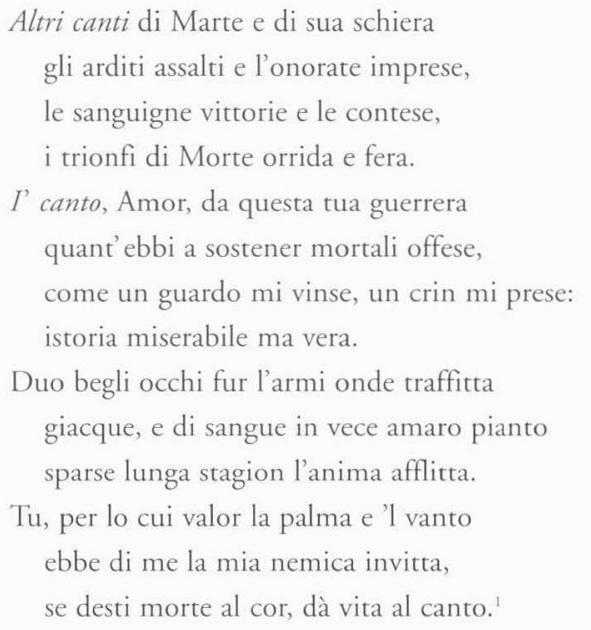

Una tale apertura era tutt altro che scontata in seno alla tradizione petrarchista. ${ }^{2}$ Palese è, fra l'altro, lo scarto rispetto alla linea Bembo-Della Casa, che proprio dalla protasi del poema eroico aveva desunto, in esordio del canzoniere, il motivo dell'invocazione alle muse. Né più significativi contatti il testo di Marino stringeva, per questo aspetto, con il modello tassiano, che nel sonetto proemiale alle rime esibiva sì un confronto fra genere epico e genere lirico, ma nei termini di un paragone ("Vere fur queste gioie e questi ardori / ond'io piansi e cantai con vario carme, I che potean agguagliare il suon de l'arme I e de gli Heroi le glorie e i casti amori»). ${ }^{3}$ Da tale precedente sembra derivare semmai il marchio di autenticità rivendicato alla vicenda amorosa, come nell'incipit di Tasso, anche al mariniano v. 8 ("istoria miserabile ma vera").

Marino si muoveva in effetti, qui e altrove, nel solco di un diverso 
classicismo. A questo proposito è utile segnalare che un probabile ipotesto del sonetto in questione è rintracciabile nel carme di Orazio ( 1,7$)$ in cui, con analoga dispositio, il poeta lasciava ad altri il compito di celebrare le famose città, riservandosi il gusto e il piacere dei paesaggi boschivi:

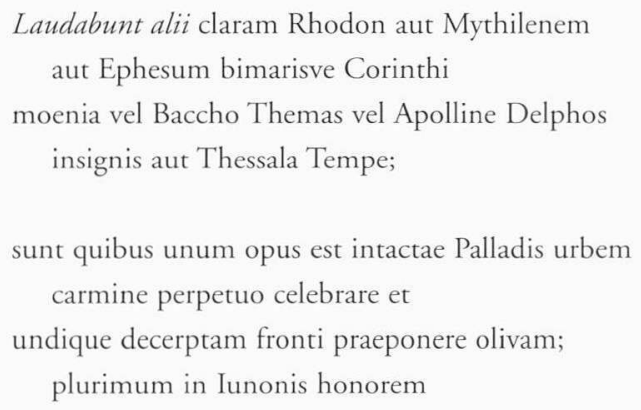

aptum dicet equis Argos ditisque Mycenas;

me nec tam patiens Lacedaemon

nec tam Larisae percussit campus opimae,

quam domus Albuneae resonantis

et praeceps Anio ac Tiburni lucus et unda mobilibus pomaria rivis...

Il confronto suggerisce l'ipotesi che Marino abbia adattato il procedimento oraziano ad un diverso contenuto, calando nello stesso schema, anziché il contrasto fra città e scena campestre, quello fra poesia epica e poesia d'amore. Orazio era poeta che egli, ovviamente, non ignorava e poteva benissimo mettere a frutto direttamente. Ciò nonostante, ritengo che alla formazione del testo mariniano abbia concorso in maniera decisiva l'esempio di un maestro della vecchia scuola napoletana come Antonio Minturno, che aveva inaugurato le proprie rime, stampate a Venezia nel 1559, con un sonetto analogamente ispirato al brano oraziano: ${ }^{4}$

I sacri fiumi, i lieti piani e i monti,

le città grandi e l'onorate prove

dican gli altri d'alcun figliuol di Giove,

o pur de' Paladin' famosi e conti.

Chi non sa Tempo e di Permesso i fonti,

Elicona, Parnaso, Olimpo e dove

fu Tebe o Troia, ond' Istro o Nilo move, 
qual fronde onora le più chiare frondi?

Io canto la divina, alma Beltate,

ignuda prima et or d'umana forma

vestita, qual non vide unq'altra etate,

che col bel sol de le sue luci amate

l'animo de' mortali adorna e 'nforma,

per farle poi nel ciel sempre beate.

Si noterà che Minturno si era mantenuto maggiormente fedele alla fonte, restando in essere, nella fronte del sonetto, la rinuncia alla celebrazione in versi di toponimi classici. Egli vi aveva inserito però, ai vv. 2-4, una presa di distanza verso le imprese di eroi antichi e moderni che anticipa quella messa ad effetto da Marino; e per di più già nel testo minturniano lo schema oppositivo era stato piegato, nella sirma, all'enunciazione di un programma di poesia amorosa. Che il ricordo oraziano passasse proprio attraverso la mediazione di Minturno conferma peraltro, oltre al rapporto fra $i$ sintagmi Altri canti (...) I' canto (Marino) e dican gli altri (...) Io canto (Minturno), specie quello che lega il secondo verso di entrambi $i$ sonetti, ove il secondo emistichio di Marino ("l'onorate imprese») risente, evidentemente, di quello di Minturno ("l'onorate prove»).

L'agnizione è tale da sollecitare una più estesa indagine su prestiti e calchi minturniani nell'opera di Marino; anche se è bene avvertire che, ad una prima ricognizione, tale presenza sembra tutt'altro che pervasiva. $\dot{E}$ significativo, ad esempio, che la serie di quattro sonetti di invocazione al Sonno (Amorose 60-63) non sembra tenere in alcun conto il sonetto minturniano O Sonno, de' mortai mirabil freno, ${ }^{5}$ ma si muove nella scia tracciata da quello, celebre, del Casa ${ }^{6}$ e ancor più si avvicina, giova qui segnalare, ad un sonetto di Guarini rivolto al Sogno.? Mi riferisco, in particolare, alla mossa iniziale del testo guariniano:

$$
\begin{aligned}
& \text { Da qual porta d'Averno apristi l'ale, } \\
& \text { col rio timor che le speranze sgombra, } \\
& \text { sogno? se sogno è quel che 'l ver m'adombra, } \\
& \text { e non, come cred' io, mostro infernale? }
\end{aligned}
$$

I contatti con l'avvio dell'ultimo componimento della quaterna di Marino sono palesi:

Da qual uscio del ciel volando uscisti, vago pittor d'imagini sì liete, 


\section{STEFANO CARRAI}

Sonno, che chiusi in placida quiete

a più sereno dì gli occhi m’apristi?

L'analogia riguarda non meno la collocazione del vocativo all'inizio del terzo verso che le parole d'esordio; e poi la relazione sinonimica di porta (Guarini) e uscio (Marino); l'antitesi, anch'essa rivelatrice di un rapporto fra i due testi, tra la provenienza infernale dell'incubo (Guarini) e l'origine celestiale del sonno che reca sogni beati (Marino); l'equivalenza d'immagine e di tempo verbale in apristi l'ale (Guarini) e in volando uscisti (Marino). E la stessa voce apristi occorre in entrambi, dal momento che compare anche, diversamente contestualizzata, al v. 4 di Marino.

Le implicazioni guariniane nella lirica di Marino non costituiscono una novità. La tessera or ora rilevata viene ad incrementare una serie di raffronti già indicati nel commento di Besomi e Martini. Acquisti di tale sorta non possono meravigliare, dunque, anche in ragione dei rapporti personali intercorsi tra $i$ due poeti, documentati entro l'epistolario mariniano fin dal 1601.' Neppure deve sorprendere tuttavia la presenza di Minturno nel sonetto proemiale delle Rime amorose. I debiti contratti dal giovane Marino, più in generale, con la generazione di Rota e di Tansillo sono stati rilevati da tempo. ${ }^{10}$ Logico è che un preciso influsso avesse esercitato un poeta che già Garcilaso, durante il suo soggiorno napoletano, aveva mostrato di conoscere e di apprezzare quale cantore, insieme con Tansillo e Bernardo Tasso, di Maria di Cardona marchesa della Padula," tanto più che Minturno aveva visto consolidarsi il proprio prestigio, non solo a Napoli, in virtù di una robusta produzione trattatistica latina e volgare. Morto vescovo nel 1573, egli era un nome illustre quando Marino cominciava a far versi e ancora quando metteva mano alla raccolta delle Amorose; anzi era stato rilanciato e quasi consacrato nell'empireo degli intendenti di poesia da Torquato Tasso, che gli aveva intitolato uno dei suoi dialoghi: Il Minturno overo de la Bellezza. ${ }^{12}$

La comune appartenenza o vicinanza alla tradizione partenopea avrà in certa misura favorito, nel caso di Tasso e di Marino, una ricezione degli scritti di Minturno. Due lettori di questo calibro sono sufficienti, ad ogni modo, a dimostrare la vitalità e l'importanza raggiunta nel secondo Cinquecento da un autore oggi generalmente negletto che, mettendo in cantiere per tempo trattati di poetica e raccolte di versi spirituali, aveva saputo interpretare come pochi altri l'età segnata dal Concilio e dal conseguente richiamo all'ordine.

Stefano Carrai 
1. Giovan Battista Marino, Rime amorose, a cura di O. Besomi e A. Martini, Modena, 1987, p. 35.

2. A tale proposito, limitatamente all'ambito del petrarchismo cinquecentesco, si ricordino almeno: F. Erspamer, Il canzoniere rinascimentale come testo o come macrotesto: il sonetto proemiale, "Schifanoia", IV (1987), pp. 109-14; G. Gorni, Il libro di poesia cinquecentesco: principio e fine, in Il libro di poesia dal copista al tipografo, a cura di M. Santagata e A. Quondam, Modena, 1989, pP. 35-41; A. Kablitz, Die Selbstimmung des petrarkistischen Diskurses im Proëmialsonett (Giovanni Della Casa-Gaspara Stampa) im Spiegel der neueren Diskussion um den Petrarkismus, "GermanischRomanische Monatsschrift", N.F., 42 (1992), pp. 381-414; M. Boaglio, Il proposito dell'imitazione. Liriche d'esordio e canzonieri petrarcheschi nel primo Cinquecento, in Teoria e storia dei generi letterari. Luoghi e forme della lirica, a cura di G. Barberi Squarotti, Torino, 1996, pp. 85-118; S. Carrai, Il canzoniere di Giovanni Della Casa dal progetto dell'autore al rimaneggiamento dell'edizione postuma, in Per Cesare Bozzetti. Studi di letteratura e filologia italiana, Milano, 1996, pp. 471-98 (in particolare pp. 473-76).

3. Cfr. Torquato Tasso, Rime d'amore, a cura di F. Gavazzeni, M. Leva, V. Martignone, introduzione di V. Martignone, Modena, 1993, p. 3.

4. Cfr. S. Carrai, Sulle rime del Minturno. Preliminari d'indagine, in Il libro di poesia dal copista al tipografo cit., pp. 215-30.

5. Cfr. Rime et Prose del Sig. Antonio Minturno, Venezia, Rampazzetto, 1559, p. 37; e G. Ferroni - A. Quondam, La "locuzione artificiosa”. Teoria ed esperienza della lirica a Napoli nell'età del manierismo, Roma, 1973, p. 307.

6. Cfr. S. Carrai, Ad Somnum. Linvocazione al Sonno nella lirica italiana, Padova, 1990, p. 66.

7. Cfr. Giovan Battista Guarini, Opere, a cura di M. Guglielminetti, Torino, $1971^{2}$, p. 28.

8. Se ne veda il regesto in Marino, Rime amorose cit., p. 206.

9. Si veda la lettera a Gaspare Salviani spedita in quell'anno da Firenze: cfr. Giovan BATtista Marino, Lettere, a cura di M. Guglielminetti, Torino, 1966, p. 28.

10. Cfr. O. Besomi, Ricerche intorno alla "Lira» di Giovan Battista Marino, Padova, 1969, p. 55.

11. Cfr. Garcilaso de la Vega, Poesías completas, edición de Á. L. Prieto de Paula, Madrid, 1989, p. 67: «Ilustre honor del nombre de Cardona, / décima moradora de Parnaso, / a Tansillo, a Minturno, al culto Taso / sujeto noble de imortal corona».

12. Per un quadro d'insieme si ricorra a E. Raimondi, Il petrarchismo nell'talia meridionale (1973), ora in Id., Rinascimento inquieto, Torino, 1994, pp. 267-306, e anche in Id., I sentieri del lettore, a cura di A. Battistini, Bologna, 1994, I, pp. 315-53. 